\title{
ESTADO DE DESARROLLO PSICOMOTOR EN NIÑOS SANOS QUE ASISTEN A UN CENTRO INFANTIL EN SANTO DOMINGO, ECUADOR
}

\author{
PSYCHOMOTOR DEVELOPMENT STATUS IN HEALTHY CHILDREN ATTENDING A CHILD CENTER \\ IN SANTO DOMINGO, ECUADOR
}

ESTADO DO DESENVOLVIMENTO PSICOMOTOR EM CRIANÇAS SAUDÁVEIS QUE PARTICIPAM

A UM CENTRO DA INFÂNCIA EM SANTO DOMINGO, EQUADOR

Jackeline Román Sacón

Pontificia Universidad Católica del Ecuador. Santo Domingo, Ecuador.

jjromans@pucesd.edu.ec

ORCID: 0000-0002-9937-113X

\section{Pamela Calle Contreras}

Pontificia Universidad Católica del Ecuador. Santo Domingo, Ecuador. ORCID: 0000-0002-4828-8037

DOI: https://doi.org/10.22235/ech.v6i2.1467

Enviado: $29 / 07 / 2017$

Aceptado: 25/10/2017

\section{RESUMEN}

El objetivo de este artículo es describir los puntajes de desarrollo psicomotor en niños y niñas que acuden un centro infantil, mediante el formulario 028 que corresponde al Test de Denver II, estandarizado internacionalmente. Se trata de un estudio con un enfoque cuantitativo de carácter descriptivo-transversal. Se reclutaron 42 niños de 18-36 meses, que acuden al centro infantil en Santo Domingo de los Tsáchilas, Ecuador, para la aplicación del test de Denver II. Se excluyeron los niños con patologías graves con impedimento para contestar el instrumento por déficit en su capacidad física y mental. Mediante los puntajes obtenidos por la aplicación del test de Denver se observó que el déficit en el Área Personal-Social se da más en los niños (20\%) que en las niñas (5\%). En el Área Motriz Fino-Adaptativo el déficit se presenta tanto en los niños (10\%) como en las niñas (9\%). En el Área de Lenguaje se observó que existe un retardo muy marcado, se presentó más en los niños (35\%) que en las niñas (18\%). Al igual se observó que el déficit en el Área Motriz Grande se da más en los niños (15\%) que en las niñas (5\%). Destaca la alta frecuencia de déficit en el desarrollo psicomotor en la población evaluada, especialmente en los niños, donde el área más significativa fue la de Lenguaje. Se recomienda la estimulación temprana y los patrones de crianza para mejorar el desarrollo del lenguaje y el desempeño de los niños en las demás áreas.

Palabras Clave: Desarrollo Infantil, Estimulación Física, Desempeño Psicomotor

\section{ABSTRACT}

The goal of this article is to describe psychomotor development scores in children who attend the children's center through Form 028, corresponding to the Internationally Standardized Denver II Test. This is a study with a quantitative approach of descriptive-transversal character. A total of 42 children aged 18-36 months were recruited to attend the Denver II test in Santo Domingo de los Tsáchilas Children's Center in Ecuador. Children with severe pathologies with impediment to answer the form due to deficiency in their physical and mental capacity were excluded. Through the scores obtained by the application of the Denver test, it was observed that the deficit in the Personal-Social Area occurs more in boys (20\%) than in girls (5\%). In the Fine Motor Skill Area, the deficit occurs in both boys $(10 \%)$ and girls (9\%). In the Language Area it was observed that there is a significant retardation: it was more present in boys (35\%) than in girls (18\%). It was also observed that the deficit in the Gross Motor Area is higher in boys (15\%) than in girls (5\%). It is significant the high frequency of deficits shown in psychomotor development in the population evaluated, especially in the language area. Early stimulation and parenting patterns are recommended to improve language development and the performance of children in other areas.

Keywords: Child Development, Physical Stimulation, Psychomotor Performance 


\section{RESUMO}

O presente estudo buscou descrever escores de desenvolvimento psicomotor em crianças que frequentam o Centro das Crianças usando o formulário de 028 correspondente ao Teste de Denver II, padronizado internacionalmente. Pesquisa quantitativa com abordagem transversal descritivo. 42 crianças com idades entre 18-36 meses que frequentam o centro infantil em Santo Domingo de los Tsáchilas, Ecuador, para a implementação do teste de Denver II foram recrutados. Crianças com doenças graves foram excluídos devido a um impedimento para atender o déficit de instrumento em sua capacidade física e mental. Usando os escores obtidos pela aplicação do teste de Denver foi observado que o déficit na área pessoal-social é mais comum em crianças (20\%) e mulheres (5\%). Na Área Motriz fina o déficit ocorre em crianças (10\%) e mulheres (9\%). Na área da linguagem foi observado que há um atraso acentuado, parecia mais filhos (35\%) do que em meninas (18\%). Como observou-se que o déficit na área Motriz Grande é mais comum em crianças (15\%) do que meninas (5\%). Destaca a alta freqüência de déficits no desenvolvimento neuropsicomotor na população estudada, especialmente em crianças. Onde a área mais significativa foi Idioma. estimulação precoce e padrões parentais é recomendado para melhorar o desenvolvimento da linguagem e desempenho das crianças em outras áreas.

Palavras-chave: Desenvolvimento Infantil, Estimulação Física, Desempenho Psicomotor

\section{INTRODUCCIÓN}

El desarrollo psicomotor (DSM) es un proceso evolutivo e integral, mediante el cual las personas van dominando progresivamente habilidades y respuestas cada vez más complejas (1). En los últimos años se han llegado a conocer mucho mejor las relaciones entre la salud, el crecimiento físico, el desarrollo psicológico y los cuidados que prodigan los padres en los primeros años de vida; el desarrollo cerebral y biológico depende de la calidad de estimulación que el niño recibe de su entorno: familia, comunidad y sociedad. La estimulación temprana es punto fundamental para partir con un desarrollo psicomotor adecuado para los niños, por lo cual la intervención temprana tiene alto impacto en el pronóstico de los niños con déficit del desarrollo (2).

Según la Organización Mundial de la Salud (OMS) anualmente mueren en el mundo casi diez millones de niñas y niños menores de cinco años; por otro lado un número casi 20 veces mayor, más de 200 millones, sobreviven pero no llegan a desarollar todas sus potencialidades humanas porque sus familias son pobres y no cuentan con nutrición, cuidados adecuados y sus oportunidades de aprender son limitadas (3). La buena nutrición, la salud, los constantes cuidados afectuosos y el estímulo en los primeros años de vida ayudan a los niños a obtener mejores resultados escolares, estar más sanos y participar en la sociedad. La Academia Americana de Pediatría define como problemas del desarrollo a todos aquellos cuadros crónicos y de inicio precoz que tienen en común la dificultad en la adquisi- ción de habilidades motoras, de lenguaje, sociales o cognitivas que provocan un impacto significativo en el progreso del desarrollo de un niño (4).

El desarrollo psicomotriz requiere de la participación activa de distintos factores, los cuales pueden beneficiar o perjudicar el adecuado desenvolvimiento del niño en el medio que lo rodea (5). Se define al desarrollo psicomotriz (DPM) como un proceso continuo que va desde la concepción a la madurez, con una secuencia similar en todos los niños, pero con un ritmo variable. Mediante este proceso el niño adquiere habilidades en distintas áreas como son: Lenguaje, Motora, Cognitiva y Personal-Social, que le permiten una progresiva independencia y adaptación al medio (6). Jean Piaget contribuyó enormemente al entendimiento del desarrollo de la inteligencia. Su visión naturalista y biológica surge de observar la interacción de los niños con su medio ambiente y de la comprensión de los procesos internos de organización y adaptación que le permiten dar un nuevo sentido al mundo que lo rodea (7). Entre los principales aportes de Piaget está el haber cambiado el paradigma niño, de un ser que recibe y acumula conocimientos en base a estímulos y refuerzos externos al estilo conductista, a un sujeto activo que construye su conocimiento desde adentro, gracias a la continua exploración del medio que lo rodea (8).

Uno de los primeros esquemas mentales que desarrolla el bebé de cuatro a ocho meses es el de objeto permanente. Piaget concibe a la inteligencia como la capacidad que tiene cada individuo de adaptarse al medio que lo rodea, adaptación que requiere del equilibrio entre los mecanismos de acomodación y asimilación (9). Cuando las experiencias de los niños sobre su entorno no encajan en su estructura mental se produce en él una situación de desequilibrio. En un primer momento, se produce una asimilación del estímulo sin que esto constituya un cambio en la estructura mental; pero posteriormente, dentro de un proceso de acomodación, se modifica la estructura para incorporar los nuevos elementos, logrando así un estado de equilibrio (10).

La motricidad refleja todos los movimientos del ser humano. Estos movimientos determinan el comportamiento motor de los niños de 1 a 6 años que se manifiestan por medio de habilidades motrices básicas, que expresan a su vez los movimientos naturales del hombre (8).

Consideramos que la motricidad es la estrecha relación que existe entre los movimientos, el desarrollo psíquico, y el desarrollo del ser humano. Es la relación que existe entre el desarrollo social, cognitivo afectivo y motriz que incide en nuestros niños como una unidad (11).

La motricidad se clasifica en motricidad fina y motricidad gruesa: la fina se refiere a las acciones que implican pequeños grupos musculares de cara, manos y pies, concretamente, a las palmas de las manos, los ojos, dedos y músculos que rodean la boca. Es la coordinación entre lo que el ojo ve y las manos tocan (12). Estos músculos son los que posibilitan la coordinación ojo-mano, abrir, cerrar y mover los ojos, mover la lengua, sonreír, soplar, hacer nudos en los cordones, agarrar un objeto, 
recortar una figura, etc. Por otro lado, la gruesa es aquella relativa a todas las acciones que implican grandes grupos musculares; en general, se refiere a movimientos de partes grandes del cuerpo del niño o de todo el cuerpo (13). Así, la motricidad gruesa incluye movimientos musculares de piernas, brazos, cabeza, abdomen y espalda, permitiendo de este modo subir la cabeza, gatear, incorporarse, voltear, andar, mantener el equilibrio, etc. (13).

El vínculo afectivo se define como un lazo de afecto filial que una persona establece con otra y que se manifiesta mediante el intento de mantener un alto nivel de proximidad con quien es objeto del apego (14). El recién nacido pone en evidencia una serie de sistemas de comportamiento que llaman la respuesta y cercanía materna, y que por tanto estimulan la creación de vínculos afectivos. Entre estos comportamientos están las expresiones faciales de agrado, interés, malhumor, disgusto y rechazo y la capacidad visomotora para seguir con la vista y fijar la mirada en objetos quietos o en movimiento (15).

Aquí importa también pensar en el lenguaje, una invención humana y el principal medio de comunicación entre las personas; los seres humanos tenemos la necesidad de relacionarnos con nuestros semejantes con diversos propósitos, para expresar necesidades, deseos, sentimientos, conocimientos e información. Cabe señalar que el lenguaje es la más grande invención porque es el principal medio de comunicación, y para los niños constituye la manera de dar a conocer sus ideas, sentimientos y poder lograr así una comunicación con las personas que se encuentran a su alrededor (7).

El artículo tiene como objetivo identificar el estado de desarrollo psicomotriz en niños del Centro Infantil, teniendo en cuenta que por lo general provienen de familias de condición socioeconómica baja, y como consecuencia muchos de ellos están privados de una estimulación adecuada que ayuda a conocer cómo pueden afectar ciertas variables para un adecuado desarrollo psicomotor.

\section{METOdOLOGÍA}

El estudio tiene un enfoque cuantitativo de carácter descriptivo-transversal. El universo está conformado por los niños que asisten al centro infantil en Santo Domingo de los Tsáchilas, Ecuador. La muestra fue conformada por preescolares de 18 a 36 meses que se encontraban asistiendo normalmente. Se excluyeron los niños con patologías graves, como los que tenían un diagnóstico conocido de enfermedad neurológica, congénita o metabólica o con impedimento para contestar el instrumento por déficit en su capacidad física y mental.

El periodo de investigación fue durante el mes de diciembre del año 2016, con una población mensual de 50 personas, de las cuales 42 se encontraban en sus capacidades físicas y mentales para la realización del test de Denver II.

La técnica de recolección fue la entrevista y el instrumento fue el formulario 028, test de Denver II formado por bloques, que consta de 125 ítems, para evaluar la evolución de acuerdo a la edad y el área de desarrollo de niños entre las dos semanas y los seis años de edad. Dicho formulario sirvió para detectar oportunamente retrasos en el Neurodesarrollo y se estandarizó como prueba de tamizaje en primer y segundo nivel de atención. El resultado de las evaluaciones realizadas permitieron obtener datos estadísticos sobre la prevalencia de niños menores de 5 años con retraso en el Neurodesarrollo transitorio y permanente; estos datos ayudaron a realizar un plan de intervención oportuna con el fin de evitar deterioro en la funcionalidad de sus actividades cotidianas (16).

\section{RESULTADOS}

En las tablas se pueden observar los resultados obtenidos en cada una de las áreas de estudio según el sexo. El déficit fue significativo, donde el mayor porcentaje se dio en los niños.

\section{Tabla 1: Estado Personal-Social de niños que acuden al Centro Infantil}

Partiendo de los resultados mediante la tabla se explica el porcentaje de niños y niñas del Centro Infantil, donde se identifica en cuál de ellos radica más el déficit en el Área Personal-Social.

Se observó que hay diferencias por sexo, donde el déficit en el Área Personal-Social se da más en los niños que en las niñas.

Tabla 1. Estado Personal-Social de niños que acuden al Centro Infantil

\begin{tabular}{|l|c|c|c|c|c|c|}
\hline OPCIONES & NIÑOS & PORCENTAJES & NIÑAS & PORCENTAJES & TOTAL NIÑOS/AS & PORCENTAJES \\
\hline NORMAL & 16 & $80 \%$ & 21 & $95 \%$ & 37 & $88 \%$ \\
\hline DUDOSO & 0 & $0 \%$ & 0 & $0 \%$ & 0 & 0 \\
\hline ANORMAL & 4 & $20 \%$ & 1 & $5 \%$ & 5 & $12 \%$ \\
\hline TOTAL & 20 & $100 \%$ & 22 & $100 \%$ & 42 & $100 \%$ \\
\hline
\end{tabular}


Tabla 2: Estado Motriz Fino-Adaptativo de niños que acuden al Centro Infantil

Mediante la tabla se explica el porcentaje del estado de desarrollo psicomotor de niños y niñas del Centro Infantil, donde se observa en cuál de ellos se inclina más el déficit en el Área Motriz Fino-Adaptativo.

Al realizar un análisis de los porcentajes en general del estudio, se observó que el déficit en el Área Motriz Fino-Adaptativo se presenta tanto en los niños como en las niñas.
Tabla 3: Estado del Área de Lenguaje de niños que acuden al Centro Infantil

Partiendo de los resultados mediante la tabla se explica el porcentaje del estado de desarrollo psicomotriz de los niños y niñas del Centro Infantil, donde se identifica en cuál de ellos radica más el déficit en el Área de Lenguaje.

Al realizar un análisis de los porcentajes en general del estudio, se observó que existe un retardo muy marcado en el Área de Lenguaje, donde se presenta más en los niños que en niñas.

Tabla 2. Estado Motriz Fino-Adaptativo de niños que acuden al Centro Infantil

\begin{tabular}{|l|c|c|c|c|c|c|}
\hline OPCIONES & NIÑOS & PORCENTAJE & NIÑAS & PORCENTAJE & TOTAL NIÑOS/AS & PORCENTAJE \\
\hline NORMAL & 18 & $90 \%$ & 20 & $91 \%$ & 38 & $90 \%$ \\
\hline DUDOSO & 0 & $0 \%$ & 0 & $0 \%$ & 0 & $0 \%$ \\
\hline ANORMAL & 2 & $10 \%$ & 2 & $9 \%$ & 4 & $10 \%$ \\
\hline TOTAL & 20 & $100 \%$ & 22 & $100 \%$ & 42 & $100 \%$ \\
\hline
\end{tabular}

Tabla 3. Estado del Área de Lenguaje de niños que acuden al Centro Infantil

\begin{tabular}{|l|c|c|c|c|c|c|}
\hline OPCIONES & NIÑOS & PORCENTAJE & NIÑAS & PORCENTAJE & TOTAL NIÑOS/AS & PORCENTAJE \\
\hline NORMAL & 13 & $65 \%$ & 18 & $82 \%$ & 31 & $74 \%$ \\
\hline DUDOSO & 0 & $0 \%$ & 0 & $0 \%$ & 0 & 11 \\
\hline ANORMAL & 7 & $35 \%$ & 4 & $18 \%$ & 42 & $100 \%$ \\
\hline TOTAL & 20 & $100 \%$ & 22 & $100 \%$ & 4 \\
\hline
\end{tabular}

Tabla 4: Estado del Área Motriz Grande de niños que acuden al Centro Infantil

Partiendo de los resultados mediante la tabla se explica el porcentaje del estado de los niños y niñas del Centro Infantil, donde se identifica en cuál de ellos radica más el déficit en el Área Motriz Grande.
Al realizar un análisis de los porcentajes en general del estudio, se observó que el déficit en el Área Motriz Grande se da más en los niños que en las niñas.

Tabla 4. Estado del Área Motriz Grande de niños que acuden al Centro Infantil

\begin{tabular}{|l|c|c|c|c|c|c|}
\hline OPCIONES & NIÑOS & PORCENTAJE & NIÑAS & PORCENTAJE & TOTAL NIÑOS/AS & PORCENTAJE \\
\hline NORMAL & 17 & $85 \%$ & 21 & $95 \%$ & 38 & $90 \%$ \\
\hline DUDOSO & 0 & $0 \%$ & 0 & $0 \%$ & 0 & $0 \%$ \\
\hline ANORMAL & 3 & $15 \%$ & 1 & $5 \%$ & 4 & $10 \%$ \\
\hline TOTAL & 20 & $100 \%$ & 22 & $100 \%$ & 42 & $100 \%$ \\
\hline
\end{tabular}




\section{DISCUSIÓN}

En nuestro estudio los puntajes de DSM, de acuerdo con el test de Denver II, fueron incrementando según la edad cronológica de los niños. Al realizar un análisis de los porcentajes en general del estudio, se observó que el déficit en las áreas se da más en los niños.

Esto se contrapone con el estudio realizado por Puente, ya que en su investigación, donde se buscaba determinar la relación entre el Estado Nutricional y el Desarrollo Psicomotriz, se obtuvo como resultado que los niños con desnutrición leve tienen problemas en el desarrollo y mediante datos recabados el mayor porcentaje de anormalidad en esta área fue en las niñas (17).

En el Área Motriz Fino-Adaptativo se presenta tanto en los niños como en las niñas, esto se diferencia con el estudio realizado por Ternera ya que en su investigación, expone las características del desarrollo motor (Control muscular, Coordinación corporal, Locomoción, Motricidad fina, Motricidad perceptiva), donde los resultados revelaron puntajes de desempeño más bajos de lo esperado para la edad. El mayor porcentaje $(38,1 \%)$ con anormalidad fue en el área Motriz Fino especialmente de la musculatura fina de brazos y manos que permite llevar a cabo tareas cada vez más complejas (18).

Al igual se observó que existe un retardo muy marcado en el Área de Lenguaje, donde de la misma forma se presenta más en los niños; esto tiene relación con el estudio de Puente, en el que se evidencia que en esta área también existe un retardo muy significativo al contrario que en el Área Motriz Grande, ya que en nuestra investigación se observó que el déficit se da más en los niños (17).

\section{CONCLUSIÓN}

El área Personal-Social de los niños y niñas se determinó como normal en un porcentaje alto, mientras que un porcentaje pequeño presentan un déficit de atención. En el Área Motor Fino-Adaptativo de los niños y niñas se estableció que un porcentaje igual presenta déficit especialmente de la musculatura fina de brazos y manos que permite llevar a cabo tareas cada vez más complejas.

En el Área de Lenguaje de los niños y niñas se determinó que existe un déficit muy marcado en esta área. En el Área Motor Grande de los niños(as) se determinó como normal en un porcentaje alto, mientras que un porcentaje menor presenta un déficit en la coordinación de la motricidad grande. La carencia de estimulación temprana probablemente sea el motivo por el cual la intervención a tiempo tiene alto impacto en el pronóstico de los niños con déficit del desarrollo.

Se recomienda la estimulación temprana en todas las áreas de desarrollo para mejorar la motricidad, gruesa y fina, y el Lenguaje, jugando con ellos, hablándoles, inventando retos juntos; de esta forma incluso se fortalecerán los vínculos.
Cómo citar este artículo: Román Sacón, J; Calle Contreras, J. Estado de desarrollo psicomotor en niños sanos que asisten a un centro infantil en Santo Domingo, Ecuador. Enfermeria (montev.). [Internet]. 2017 Dic. [citado xxxxx]; 6 (2) 39-44. Disponible en: https://doi.org/10.22235/ech.v6i2.1467

\section{REFERENCIAS BIBLIOGRÁFICAS}

1. Schönhaut, ,L, Schonstedt, M., Álvarez, J., Salinas, P., \& Armijo, I. Desarrollo psicomotor en niños de nivel socioeconómico medio-Alto. Rev. chil. pediatr . [Internet]. 2010. [Citado 3 Nov 2016] ; 81(2), 123-128. Disponible en: http://www.scielo.cl/scielo.php?pi$\mathrm{d}=$ S0370-41062010000200004\&script=sci_arttext

2. Pando, M., Aranda, C., Amezcua, M., Salazar, J., Torres, T. (2004). Estimulación temprana en niños de 4 años de familias marginadas. Recuperado el 13 enero 2017 de http:// www.medigraphic.com/pdfs/pediat/sp-2004/sp046c.pdf

3. Organización Mundial de Salud. Desarrollo [Internet]. [Citado 23 Oct 2016] Disponible en: http://www.who.int/ maternal_child_adolescent/topics/child/development/es

4. American Academy of Pediatrics Committee on Children with Disabilities: The pediatrician's role in development and implementation of an Individual Education Plan (IEP) and/ or an Individual Family Service Plan (IFSP). Pediatrics 1999; 104: 124-7. [Internet]. [Citado 24 Oct 2016] Disponible en: http://www.scielo.cl/scielo.php?script=sci_nlinks\&re$f=6856707 \&$ pid $=$ S0370-4106201000050000200001\&ln$\mathrm{g}=\mathrm{es}$

5. Moran Aguilar, V., \& Robles, A. L. M. Proceso de enfermería. Uso de lenguajes NANDA, NIC y NOC. Modelos referenciales. México, D.F. : Editorial Trillas; 2010.

6. AEPNYA. Protocolo del niño sano. [Internet]. [Citado 26 Nov 2016]. Disponible en: https://www.aeped.es/sites/ default/files/documentos/nino_sano_0-2_anos.pdf

7. Medina, A. La estimulación temprana disponible (2002). Recuperado el 13 enero 2017 de http://www.medigraphic. com/pdfs/fisica/mf-2002/mf02-2_4i.pdf

8. Madrona, P. G., Contreras Jordán, O. R,. Gómez Barreto, I. Habilidades motrices en la infancia y su desarrollo desde una actividad física animada. RIE [Internet]. 2008. [Citado 20 Nov 2016]; 47 Disponible en: http://rieoei.org/ rie47a04.htm

9. Coutiño, LB. Desarrollo psicomotriz. Rev Mex Med Fis Rehab 2002; 14 (2-4)

10. Universidad Politécnica de Madrid. PINEF. Desarrollo motor. [Internet]. 2014 [Citado 20 Nov 2016] Disponible en: http://futbolcarrasco.com/wp-content/uploads/2014/08/ futbolcarrascoinef2curso7.pdf

11. Cancho Candela, R.. Desarrollo psicomotor del Niño. [Internet]. 2015 [Citado 20 Nov 2016] Disponible en: Disponible en: http://ceipgarciaquintana.centros.educa.jcyl.es/ sitio/upload/DESARROLLO_PSICOMOTOR_I.pdf

12. Pérez, R. Desarrollo Psicomotor en la Infancia. Madrid: Ideaspropias; 2015. 
13. Maganto, C. Desarrollo físico y psicomotriz en la etapa infantil. [Internet]. 2015. [Citado 3 Ene 2017]; Disponible en: http://webcache.googleusercontent.com/search?q=cache:S9TUwjNZo-oJ:www.sc.ehu.es/ptwmamac/Capi_libro/38c. pdf $+\& c d=1 \& h l=e s \& c t=c l n k \& g l=e c$

14. Penton Hernández, B. (2015). Motricidad fina en la etapa infantil. Disponible en: http://webcache.googleusercontent.com/search?q=cache:VN5k5LcPepsJ:ardilladigital. com/DOCUMENTOS/EDUCACION\%2520ESPECIAL/PSICOMOTRICIDAD\%2520-\%2520FISIOTERAPIA/CUALIDADES\%2520MOTRICES/Motricidad\%2520fina\%2520en\%2520la\%2520etapa\%2520infantil\%2520-\%2520Penton\%2520-\%2520art. $\mathrm{pdf}+\& \mathrm{~cd}=1 \& \mathrm{hl}=\mathrm{es} \& \mathrm{ct}=\mathrm{clnk} \& \mathrm{gl}=\mathrm{ec}$

15. Moreno, J. El aprendizaje en el juego motriz en la infancia. Facultad de Educación Universidad de Murcia. [Internet]. 2013. [Citado 3 Ene 2017]; Disponible en: http://webcache. googleusercontent.com/search?q=cache:IwWBVGdXr9U-
J:www.um.es/univefd/juegoinf.pdf $+\& c d=1 \& h l=e s \& c t=c l-$ $\mathrm{nk \& gl}=\mathrm{ec}$

16. Ministerio de Salud Pública. (2015). Instrumento para el Ilenado del Formulario 028 Test de Denver II. MSP: Quito; Ecuador

17. Puente, E. Relación existente entre el estado nutricional y el desarrollo psicomotriz de los niños de 4 a 5 años de la escuela Santiago Cantos Cordero (Tesis Doctoral). Escuela Politécnica del Ejército. Quito, Ecuador). Recuperado el 7 de noviembre del 2016 de, http://repositorio.espe.edu. ec/bitstream/21000/5427/1/T-ESPE-033358.pdf

18. Ternera, L. A. C. Relación entre el desarrollo personal social y los procesos evolutivos vinculados con el aprendizaje escolar en las áreas del lenguaje y la cognición. Psicogente, [Internet]. 2010. [Citado 3 Ene 2017]; 13(10). Disponible en: http://publicaciones.unisimonbolivar.edu.co/rdigital/ ojs/index.php/psicogente/article/view/449/446 